\title{
Managementgrundlagencurriculum für Assistenzärztinnen und Assistenzärzte in Spitälern 2008
}

Das Weiterbildungscurriculum ist ein kompakter Kurs (total 6 Tage) über Grundlagen des Managements, das Zusammenspiel von Arbeitsprozessen und ärztlichen Funktionen, zur Kommunikation und Kooperation in Organisationen, Qualitätsentwicklung, rechtlichen und betriebswirtschaftlichen Fragen sowie zum Selbstmanagement und zur Laufbahnplanung.

Das Weiterbildungscurriculum ist einzigartig und spezifisch auf Anforderungen und Bedürfnisse von Assistenzärztinnen und -ärzten mit dem Ziel einer Spitalarzttätigkeit zugeschnitten und wird vom College-M im Auftrag der FMH durchgeführt und vom VSAO unterstützt.

\section{Lernziele}

- Die wichtigsten Entwicklungen und deren Bedeutung im Gesundheitssystem kennen;

- ein Verständnis von Management, seinen Entwicklungen, Werkzeugen und Vorgehensweisen entwickeln;

- grundlegende Dimensionen des Führens erkennen unter Berücksichtigung gesundheitsökonomischer Aspekte und Entwicklungen;

- die wichtigsten Qualitätskonzepte und Gesetzesgrundlagen im Gesundheitssystem kennen;

- vertieftes Verständnis des Zusammenspiels verschiedener Funktionen und Prozesse in (Spital-)Organisationen;

- die Bedeutung der Selbstführung und der Laufbahnplanung kennen;

- grundlegendes Wissen um rechtliche und betriebswirtschaftliche Fragen.

\section{Teilnehmende}

Das Managementgrundlagencurriculum richtet sich an Assistenzärztinnen und -ärzte in Spitälern. Die einzelnen Curriculumgruppen bestehen aus 16-20 Teilnehmenden. Sie werden von ausgewiesenen Trainern geleitet und durch Lernpartnerschaften der Teilnehmenden unterstützt.

\section{Programmaufbau}

Das Weiterbildungscurriculum ist als Entwicklungs- und Lernprozess gestaltet und gliedert sich in zeitlich und thematisch getrennte Weiterbildungselemente. Die einzelnen Themen bzw. Elemente sind eng koordiniert und verknüpfen sich mit der beruflichen Funktion der Teilnehmenden. Die Module Evidence-based Medicine und Ökonomie werden in Form von E-Learning durchgeführt.

\section{Programmleitung}

- Daniel Germann, Dr. med., Mitglied der Geschäftsleitung Spitalregion St. Gallen Rorschach;

- Thomas Lempert, lic. theol., Psychotherapeut, Organisationsberater;

- Jana Faehnrich, Dr. med., MHA, Organisationsberaterin, Dozentin College-M;

- Hansjürg Lusti, Dipl. Psych., Systemtherapeut, Supervisor, Organisationsberater.

Die Seminare werden grösstenteils im CoTeaching geführt.

\section{Daten und Kursorte}

\section{Kurs 1/2008}

- Element 1: 11./12.4.2008, Bern

- Element 2: 23./24.5.2008, Zürich

- Element 3: 20./21.6.2008, Bern

- Element 4: 11./12.7.2008, Bern

Kurs 2/2008

- Element 1:22./23.8.2008

- Element 2: 26./27.9.2008

- Element 3: 7./8.11.2008

- Element 4: 12./13.12.2008

\section{Preis}

Der Unkostenbeitrag der Teilnehmenden beträgt Fr. 390.-.

\section{Informationen und Anmeldungen}

Detaillierte Informationen finden Sie unter: www.college-m.ch. 